\title{
Prevalence of Metabolic Syndrome in Iraqi Patients with Rheumatoid Arthritis
}

\author{
Dr. Zina Hasan Abdul-Qahar ${ }^{1}$, Dr. Mohammad Hadi Al-Osami ${ }^{2}$, \\ Dr. Firas Znki Al-Asady ${ }^{3}$ \\ 1(Department of Biochemistry, College of Medicine/University of Baghdad, Iraq) \\ 2(Department of Medicine, College of Medicine/University of Baghdad, Iraq) \\ 3(Karbala Health Directorate, Iraq)
}

\begin{abstract}
Background: Rheumatoid arthritis is a systemic autoimmune disease characterized by chronic joint inflammation and subsequent destruction. It also associates with excessive cardiovascular morbidity and mortality similar to metabolic syndrome which reflects a clustering of classical cardiovascular risk factors. This study is designed to investigate the Prevalence of Metabolic syndrome in rheumatoid arthritis patients and possible association to disease related risk factors. Material and Methods: The subjects were 203 patients with rheumatoid arthritis diagnosed according to the American College of Rheumatology criteria while metabolic syndrome was defined according to National Cholesterol Education Program criteria. Results: The overall prevalence of metabolic syndrome in rheumatoid arthritis patients was $51.2 \%$; being higher in women than men. Women with metabolic syndrome had a higher prevalence of Cardiovascular disease than those without metabolic syndrome $(p=0.004)$. Significant differences were observed in traditional cardiovascular disease risk factors which are common in rheumatoid arthritis with metabolic syndrome $(p<0.001)$. Conclusion: The prevalence of metabolic syndrome is high in patients with rheumatoid arthritis. The correlation of rheumatoid arthritis disease with metabolic syndrome suggests that the increased prevalence of Cardiovascular disease in patients with rheumatoid arthritis may be attributed to the inflammatory burden of the disease in addition to traditional Cardiovascular disease risks of metabolic syndrome.
\end{abstract}

Keywords: Atherosclerosis, body mass index, cardiovascular disease, hypertension, metabolic syndrome, rheumatoid arthritis, rheumatoid factor.

\section{Introduction}

Rheumatoid Arthritis (RA) is a systemic disease characterized by massive joint destruction and deformity; may be accompanied by extra-articular manifestations in many vital organs. The disease leads to increased morbidity and mortality especially if associated with other diseases like Metabolic Syndrome (MetS). ${ }^{[1,2]}$

Metabolic syndrome (MetS) includes many classical cardiovascular diseases (CVD) risk factors including insulin resistance, central obesity, elevated blood pressure, high triglyceride (TG) levels and low levels of high density lipoprotein (HDL), it is diagnosed when 3 or more of the National Cholesterol Education Program III (NCEP III) criteria are present ${ }^{[3,4]}$.

The prevalence estimates of RA are between $0.5 \%-1.0 \%$ and may reach to more than $5 \%$ in some populations ${ }^{[2]}$. Definite RA was observed in $1 \%$ in population samples in Iraq ${ }^{[5]}$.

Theories suggesting an inflammatory basis for atherosclerosis have led many researchers to study the relationship between systemic inflammatory conditions such as RA and the risk of coronary heart disease. Patients with RA appear to have a higher risk of coronary heart disease morbidity and mortality; the same applies for MetS. ${ }^{[6,7]}$

Although several studies have established the increased risk for CVD and the increased prevalence of insulin resistance in patients with rheumatoid arthritis, the overall prevalence of the MetS, a more global indicator of the risk of CVD, have not been studied. This is important in view of the increased morbidity and mortality from $\mathrm{CVD}^{[8]}$.

The aim of this study is to assess the prevalence of Metabolic Syndrome in Rheumatoid Arthritis patients identifying risk factors for Cardiovascular Diseases in these patients.

\section{Material \& Methods}

This study was conducted on 203 patients with RA meeting the American College of Rheumatology Criteria for the classification of rheumatoid arthritis. All patients were seen in the Department of Rheumatology in Baghdad Teaching Hospital, a tertiary referral center in Iraq between October 2009 and July 2010. 
The study was granted full ethical approval from the local ethics committee and all patients gave their informed written consent prior to commencement of the study.

\section{Assessment of the metabolic syndrome:}

The metabolic syndrome was classified by NCEP III criteria ${ }^{[3]}$ : fasting plasma glucose of at least 6.1 $\mathrm{mmol} / \mathrm{L}$ or on drug treatment for elevated blood glucose, systolic blood pressure of at least 130 and diastolic blood pressure at least $85 \mathrm{~mm} \mathrm{Hg}$ or on drug treatment for elevated blood pressure, TG at least $1.7 \mathrm{mmol} / \mathrm{L}$ or drug treatment for elevated TG, HDL in males of less than $1.0 \mathrm{mmol} / \mathrm{L}$ and in females of less than $1.3 \mathrm{mmol} / \mathrm{L}$ or on drug treatment for a low HDL, and waist circumferences in males of greater than $102 \mathrm{~cm}$ and in females of greater than $88 \mathrm{~cm}$, with the MetS defined as the presence of at least three of these criteria.

All participants underwent a thorough baseline evaluation including a detailed review of their medical history, physical examination, and contemporary assessments of basic demographics: (age, gender, height, weight, body mass index (BMI), waist circumference and smoking status). A full medical history including history of hypertension, CVD; including myocardial infarction and angina pectoris, family history of CVD, drugs taken and use of tobacco. The waist circumference was measured at the umbilical level according to the WHO guidelines, with the person breathing silently ${ }^{[9]}$. Blood pressure measurement was done by using mercury sphygmomanometer in either arm when the patient relaxed in sitting position or the arm at the level of the heart.

\section{Laboratory tests:}

Baseline blood samples were obtained from each patient and were analyzed in the same laboratory. Blood samples were taken in the morning after 10 hours of overnight fasting. Blood tests included: fasting lipid profile: [total cholesterol (TC), high density lipoprotein (HDL), low density lipoproteins (LDL) and triglycerides $(\mathrm{TG})]$, rheumatoid factor, and fasting glucose.

\section{Statistical analysis:}

The Statistical Package for Social Sciences-version 17 (SPSS-v17) was used for data input and analysis. The results were reported as mean \pm standard deviation (mean $\pm \mathrm{SD}$ ). The difference between normally distributed continuous variables was examined by t-test. Findings with $\mathrm{P}$ value equal to or less than 0.05 were considered statistically significant.

\section{Results}

Among the 203 patients with RA, 162 (79.8\%) were women and 41 (20.2 \%) were men, with a mean (SD) age of $46.9 \pm 11.5$ years. The overall prevalence of MetS was $51.2 \%$ in patients with RA, being $29.3 \%$ in male patients and $56.8 \%$ in female patients.

The important cardiovascular risk factors among patients with RA were present for hypertension (54.6 $\%$ ), and low HDL (72.5\%). The frequency of raised glucose levels or hypertriglyceridaemia was not significant. Among patients with RA, women with MetS had a higher prevalence of CVD than those without MetS (19.6\% versus $4.3 \%, p=0.004)$. Patients with MetS had higher Body Mass Index (BMI) than those without MetS $(31.2 \pm 5.2$ versus $25.3 \pm 5.0 \mathrm{~kg} / \mathrm{m} 2, \mathrm{p}<0.001)$. Significant differences were observed in traditional CVD risk factors such as advance age, high LDL levels and hypercholesterolaemia which are common in RA with MetS $(\mathrm{p}<0.001)$. History of smoking was no different among RA patients with or without MetS. Table (1).

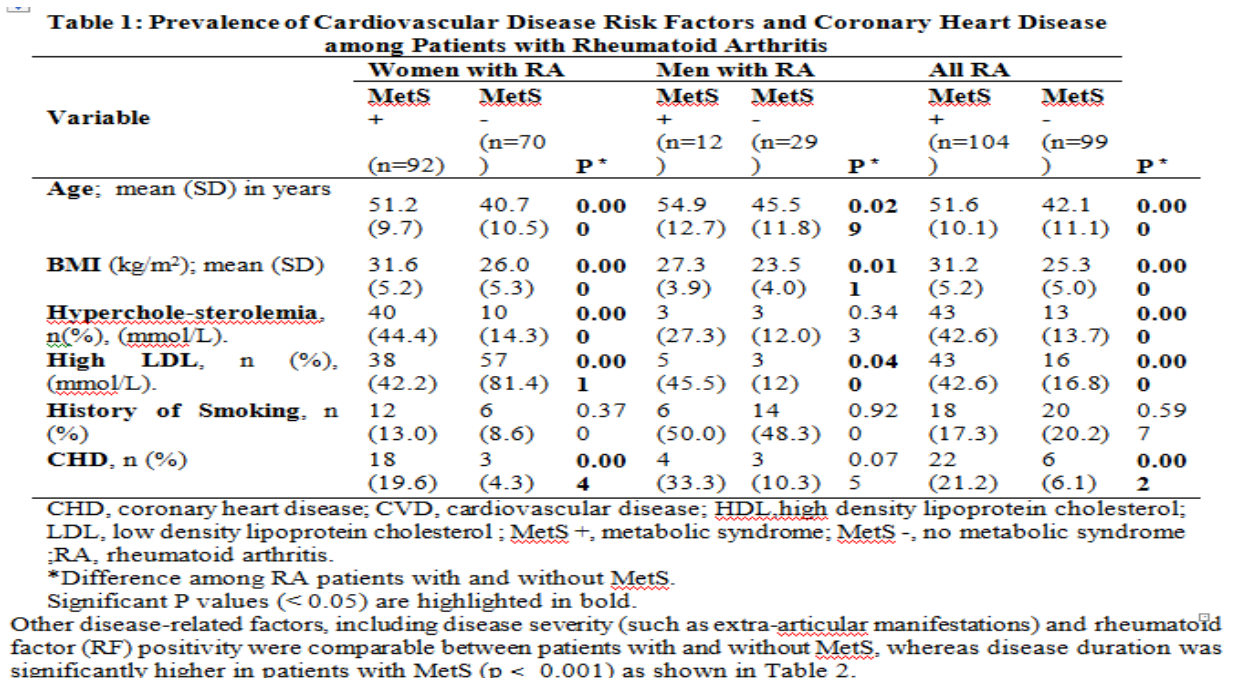


Prevalence of Metabolic Syndrome in Iraqi Patients with Rheumatoid Arthritis

Table 2: Disease characteristics among patients with Rheumatoid Arthritis to the presence of Metabolic Syndrome

\begin{tabular}{|c|c|c|c|c|c|c|c|c|c|}
\hline \multirow[b]{2}{*}{ Variable } & \multicolumn{3}{|c|}{ Women with RA } & \multicolumn{3}{|c|}{ Men with RA } & \multicolumn{3}{|c|}{ All with RA } \\
\hline & $\begin{array}{l}\text { MetS + } \\
\mathrm{n}=92 \\
(\%)\end{array}$ & $\begin{array}{l}\text { MetS- } \\
\mathrm{n}=70 \\
(\%)\end{array}$ & $\mathrm{P}^{*}$ & $\begin{array}{l}\text { MetS }+ \\
\mathrm{n}=12 \\
(\%)\end{array}$ & $\begin{array}{l}\text { MetS- } \\
\mathrm{n}=29 \\
(\%)\end{array}$ & $\mathrm{P}^{*}$ & $\begin{array}{l}\text { MetS }+ \\
\mathrm{n}=104 \\
(\%)\end{array}$ & $\begin{array}{l}\text { Mets- } \\
\mathrm{n}=99 \\
(\%)\end{array}$ & $\mathrm{P*}$ \\
\hline RF positivity, $\mathrm{n}(\%)$ & $\begin{array}{c}53 \\
(62.4)\end{array}$ & $\begin{array}{c}41 \\
(61.2)\end{array}$ & 0.884 & $\begin{array}{c}9 \\
(75.0)\end{array}$ & $\begin{array}{c}19 \\
(70.4)\end{array}$ & 1.000 & $\begin{array}{c}62 \\
(63.9)\end{array}$ & $\begin{array}{c}60 \\
(63.8)\end{array}$ & 0.990 \\
\hline $\begin{array}{l}\text { RA duration, } \\
\text { years, mean (SD) }\end{array}$ & $\begin{array}{l}51.2 \\
(9.7)\end{array}$ & $\begin{array}{c}40.7 \\
(10.6)\end{array}$ & 0.000 & $\begin{array}{c}54.9 \\
(12.7)\end{array}$ & $\begin{array}{l}45.5 \\
(11.8)\end{array}$ & 0.029 & $\begin{array}{c}51.6 \\
(10.1)\end{array}$ & $\begin{array}{c}42 \\
(11.1)\end{array}$ & 0.000 \\
\hline $\begin{array}{l}\text { Extra-articular } \\
\text { manifestations, n } \\
(\%)\end{array}$ & $\begin{array}{c}33 \\
(37.5)\end{array}$ & $\begin{array}{c}23 \\
(32.9)\end{array}$ & 0.544 & $\begin{array}{c}2 \\
(16.7)\end{array}$ & $\begin{array}{c}4 \\
(13.8)\end{array}$ & 0.813 & $\begin{array}{c}35 \\
(35.0)\end{array}$ & $\begin{array}{c}27 \\
(27.3)\end{array}$ & 0.239 \\
\hline
\end{tabular}

\section{Discussion}

Several studies concerning RA described the demographic criteria of RA patients. In this study; mean age of patients was in the fifth decade; this is in accordance with other study which mentioned that RA affects usually people above 40 years old.$^{[10]}$ This is due to many reasons that depress immunity as stress, thymic depression, and longer duration of exposure to environmental antigens that cause stimulation of auto-reactive immune cells. ${ }^{[11]}$

This study shows that females are more predominant for RA than males with a ratio of 3:1 and this agrees with previous studies done in Iraq conducted by Al-Rawi et al in 1977 and 1978. ${ }^{[5,12]}$ this female predominance in Iraq could be related to the higher number of females compared to males due to several wars, and it could be caused by hormonal factors such as estrogen which affects the function of T-lymphocytes. ${ }^{[13]}$

This study confirmed that the overall prevalence of MetS is high in RA. We have noted that five other studies have commented on the prevalence of the MetS in patients with RA, reporting prevalence rates ranging from 14 $\%$ to $45.3 \%{ }^{[14-18]}$. Such diversity can be explained by differences in the baseline characteristics, disease characteristics and definitions used.

Overall, we have reported significantly higher prevalence rates according to the NCEP 2001 as compared with other investigators. No similar study on the prevalence of MetS among Iraqi rheumatoid patients was found.

Also we found that the prevalence of MetS was significantly higher in women with RA than in men specifically in the age groups $<40$ and $\geq 60$ year. These results differ from previous studies on the prevalence of MetS done by Chung et al ${ }^{[14]}$, Karvounaris et al ${ }^{[16]}$ and Toms et al ${ }^{[18]}$ which showed the same prevalence rate between men and women. The male to female differences reported in the present study were also observed in the general population, where age-matched females have been reported to have significantly higher rates of the MetS (Ford et $a l^{[19]}$ ). The association of MetS with older age is expected and many studies support this finding $[14,16,18]$

When RA disease-related factors were examined for possible association with the presence of MetS, a significant association was found between long disease duration and increased number of criteria of MetS in agreement with previous studies ${ }^{[20,21]}$ whereas in previous studies done by Karvounaris et al ${ }^{[16]}$ and MaraditKremers et $a l^{[7,22]}$ showed no significant relation between disease duration and risk of CVD in patients with RA. Other disease-related factors, such as extra-articular manifestations and rheumatoid factor sero-positivity did not significantly associate with the presence of MetS which is compatible to the study of Karvounaris et al ${ }^{[16]}$. Furthermore, patients with MetS showed a trend for higher ESR than those without the syndrome.

Regarding distribution of MetS components in RA patients; this study showed significant increase in blood pressure and low HDL level in women with RA; this finding is corresponding to previous but not all studies ${ }^{[16]}$. Karvounaris et al showed significant increase in arterial blood pressure and waist circumference among control groups, low HDL in RA groups with no significant changes regarding fasting glucose level and triglycerides.

The data produced in the present study showed a significant high prevalence of traditional CVD risk factors and Coronary heart diseases among RA patients with MetS (increasing age, hypercholesterolemia, high LDL level, high BMI and personal history of CVD) making patients with RA at increased risk for developing CVD. Patients with RA are at increased risk for CVD, which account for about half of all deaths in these patients ${ }^{[7]}$. This is attributed to accelerated atherosclerosis as a result of both systemic inflammatory burden and the high prevalence of traditional risk factors for CVD. ${ }^{[8,9]}$ It has been proposed that the increased risk of CVD may precede the clinical onset of RA, and that there is a preclinical phase of RA during which inflammatory activity and serological disturbances occur ${ }^{[6,7,20,23]}$. 


\section{Conclusion}

MetS frequency was high in RA patients and high prevalence of cardiovascular risk factors in RA suggests that inflammatory processes play a notable role in the development of CVD, and indicates that tight control of systemic inflammatory activity and CVD modifiable risk factors should be recommended in RA patients.

\section{References}

[1]. Karl E, Eugen F, and Gerd B: The Serological diagnosis of Rheumatoid Arthritis. Deutsch Arzteblatt International 2009; 106(10): 159-63.

[2]. Waldenburger JM, Firestein GS. Rheumatoid arthritis. In: Klippel JH, Stone JH, Crofford LJ, and White PH, editors. Primer on the Rheumatic Disease, $13^{\text {th }}$ ed. New York, USA: Springer Science and business media, 2008. P.122- 32.

[3]. Executive Summary of the Third Report of The National Cholesterol Education Program (NCEP) Expert Panel on Detection, Evaluation, And Treatment of High Blood Cholesterol In Adults (Adult Treatment Panel III). JAMA 2001; 285:2486-97.

[4]. Implications of recent clinical trials for the National Cholesterol Education Programme Adult Treatment Panel III guidelines. Circulation 2004; 110:227-39.

[5]. Al-Rawi ZS, Alazzawi AJ, Alajili FM: Rheumatoid arthritis in population samples in Iraq. Ann Rheum Dis 1978; 37 : 73-5.

[6]. Da Cunha VR, Brenol CV, Brenol JCT, Fuchs SC, Arlindo EM, Melo IMF, et al: Metabolic syndrome prevalence is increased in rheumatoid arthritis patients and is associated with disease activity. Scand J Rheumatol 2012; 41: 186-191.

[7]. Maradit-Kremers H, Crowson CS, Nicola PJ, Ballman KV, RogerVL, Jacobsen SJ, et al: Increased unrecognized coronary heart disease and sudden deaths in rheumatoid arthritis: A population-based cohort study. Arth \& Rheum 2005 52(2): $402-411$.

[8]. Wolfe F, Freundlich B, Straus WL: Increase in cardiovascular and cerebrovascular disease prevalence in rheumatoid arthritis. $J$ Rheumatol 2003; 30: 36-40.

[9]. WHO Expert Committee. Physical status: the use and interpretation of anthropometry. WHO Tech Rep Ser 1995; 854:424-38.

[10]. Alamanos Y, Voulgari PV, and Doros AA: Incidence and prevalence of rheumatoid arthritis based on 1987 American College of Rheumatology criteria: A systematic review. Semin Arthritis Rheum 2006; 36(3): 182-88.

[11]. Kotzin BL: The role fB cells in the pathogenesis of Rheumatoid Arthritis. J Rheumatol Suppl. 2005; 73:14-17.

[12]. Al Rawi ZS, Al Shackarchi HA, Marjana NH, Hart FD: Rheumatoid Arthritis in Iraq. Rheumatology 1977; 16 (2): $128-132$.

[13]. Ahmed A, Dauphinee MJ, Talal N: Effects of short term administration of sex hormones on normal and autoimmune mice. $J$ Immunol 1985; 134 (1): 204-210.

[14]. Chung CP, Oeser A, Solus JF, Avalos I, Gebretsadik T, Shintani A, et al: Prevalence of the metabolic syndrome is increased in rheumatoid arthritis and is associated with coronary atherosclerosis. Atherosclerosis 2008; 196:756-63.

[15]. Zonana-Nacach A, Santana-Sahagún E, Jiménez-Balderas FJ, and Camargo-Coronel A: Prevalence and factors associated with metabolic syndrome in patients with rheumatoid arthritis and systemic lupus erythematosus. J Clin Rheumatol 2008; 14(2): 74-77.

[16]. Karvounaris SA, Sidiropoulos PI, Papadakis JA, Spanakis EK, Bertsias GK, Kritikos HD, et al: Metabolic syndrome is common among middle-to-older aged Mediterranean patients with rheumatoid arthritis and correlates with disease activity: a retrospective, cross-sectional, controlled, study. Ann Rheum Dis 2007; 66(1): 28-33.

[17]. Dessein PH, Tobias M, Veller MG: Metabolic syndrome and subclinical atherosclerosis in rheumatoid arthritis. J Rheumatol 2006; 33(12):2425-32

[18]. Toms TE, Panoulas VF, John H, Douglas KMJ, and Kitas GD: Methotrexate therapy associates with reduced prevalence of the metabolic syndrome in rheumatoid arthritis patients over the age of 60- more than just an anti-inflammatory effect? A cross sectional study. Arthritis Res Ther 2009; 11(4):R110.

[19]. Ford ES, Giles WH, Dietz WH: Prevalence of the metabolic syndrome among US adults. JAMA 2002; 287(3):356-9.

[20]. Myasoedova E, Davis III JM, Crowson CS, RgerVL, Karon BL, Borgeson DD, et al: Brief Report: Rheumatoid Arthritis Is Associated with Left Ventricular Concentric Remodeling: Results of a Population-Based Cross-Sectional Study. Arthritis Rheum 2013; 65(7): 1713-1718.

[21]. Baker JF, Mehta NN, Baker DG, Toedter G, Shults J, Von Feldt JM, et al: Vitamin D, Metabolic Dyslipidemia, and Metabolic Syndrome in Rheumatoid Arthritis. Am J Med 2012; 125: 1036.e9-1036.e15.

[22]. Maradit-Kremers H, Nicola PJ, Crowson CS, Ballman KV, and Gabriel SE: Cardiovascular death in rheumatoid arthritis: a population-based study. Arthritis Rheum 2005; 52(3):722-32.

[23]. Ormseth MJ, Lipson A, Alexopoulos N, Hartlage GR, Oeser AM, Bian A, Gebretsadik T, et al: Epicardial adipose tissue is associated with cardiometabolic risk and the metabolic syndrome in patients with rheumatoid arthritis. Arthritis Care Res 2013; DOI: $10.1002 /$ acr. 22027 . 